This item was submitted to Loughborough's Research Repository by the author.

Items in Figshare are protected by copyright, with all rights reserved, unless otherwise indicated.

\title{
Intermittency route to chaos and broadband high-frequency generation in semiconductor superlattice coupled to external resonator
}

\section{PLEASE CITE THE PUBLISHED VERSION}

http://dx.doi.org/10.1103/PhysRevE.92.022911

\section{PUBLISHER}

(C) American Physical Society

\section{VERSION}

VoR (Version of Record)

\section{PUBLISHER STATEMENT}

This work is made available according to the conditions of the Creative Commons Attribution-NonCommercialNoDerivatives 4.0 International (CC BY-NC-ND 4.0) licence. Full details of this licence are available at: https://creativecommons.org/licenses/by-nc-nd/4.0/

\section{LICENCE}

CC BY-NC-ND 4.0

\section{REPOSITORY RECORD}

Hramov, Alexander E., V.V. Makarov, V.A. Maximenko, Alexey A. Koronovskii, and Alexander G. Balanov. 2019. "Intermittency Route to Chaos and Broadband High-frequency Generation in Semiconductor Superlattice Coupled to External Resonator”. figshare. https://hdl.handle.net/2134/19045. 


\title{
Intermittency route to chaos and broadband high-frequency generation in semiconductor superlattice coupled to external resonator
}

\author{
Alexander E. Hramov, ${ }^{1,2, *}$ Vladimir V. Makarov, ${ }^{1,2}$ Vladimir A. Maximenko, ${ }^{1,2}$ \\ Alexey A. Koronovskii, ${ }^{1,2}$ and Alexander G. Balanov ${ }^{1,3, \dagger}$ \\ ${ }^{1}$ Research and Education Center 'Nonlinear Dynamics of Complex Systems', Saratov State Technical University, Saratov 410054, Russia \\ ${ }^{2}$ Faculty of Nonlinear Processes, Saratov State University, Astrakhanskaja 83, Saratov 410012, Russia \\ ${ }^{3}$ Department of Physics, Loughborough University, Loughborough LE11 3TU, United Kingdom
}

(Received 22 April 2015; published 19 August 2015)

\begin{abstract}
We investigate the onset of broadband microwave chaos in the miniband semiconductor superlattice coupled to an external resonator. Our analysis shows that the transition to chaos, which is confirmed by calculation of Lyapunov exponents, is associated with the intermittency scenario. The evolution of the laminar phases and the corresponding Poincare maps with variation of a supercriticality parameter suggest that the observed dynamics can be classified as type I intermittency. We study the spatiotemporal patterns of the charge concentration and discuss how the frequency band of the chaotic current oscillations in semiconductor superlattice depends on the voltage applied.
\end{abstract}

DOI: 10.1103/PhysRevE.92.022911

PACS number(s): 05.45.-a, 73.21.-b, 72.20.Ht

\section{INTRODUCTION}

Nowadays semiconductor superlattices (SLs) [1-4] are considered as promising elements for sub- $\mathrm{THz}$ and $\mathrm{THz}$ electronics $[5,6]$. Being formed by several alternating layers of different semiconductors, they provide a unique quantum mechanical environment [3,7], which can be utilized for generation [8,9], amplification [10,11], and detection of highfrequency electromagnetic signals $[12,13]$. In this respect, the effects of the external resonator on high-frequency current generation in SLs is of great research interest from both fundamental and practical points of view [6] for a number of reasons. First, at high frequencies it is impossible to avoid the parasitic capacitances and inductances in the elements enclosing the SL (wires, contacts, electrodynamic structures), which can significantly influence the charge transport in the device $[14,15]$.

Second, the external resonant system is widely used for tuning and enhancing the characteristics of the high-frequency generation [16]. Finally, coupling of the generating device to an external electrodynamic structure sometime leads to emergence of new unexpected phenomena, including bistability [17], self-pulsations [18,19], and appearance of chaos [20], which is unfeasible in the isolated devices.

The possibility to generate high-frequency (up to few GHz) chaos in miniband and weakly coupled SLs was previously demonstrated in both theory [21-24] and experiment [25-28]. These findings have opened wide perspectives for using SL devices in a number of key modern technologies including fast random number generation [27,29], which is crucially important in chaos-based communication systems [30-32] and cryptography [33]. Certain progress in the development of true random bit generators for applications to secure communications was achieved by using weakly coupled superlattices [27] and chaotic semiconductor lasers [29,34].

\footnotetext{
*hramovae@gmail.com

†a.balanov@lboro.ac.uk
}

Recently we discovered that a resonant electrodynamic system connected to an SL can drive the latter to generate chaotic current oscillations, even if the SL alone is unable to generate chaos [20]. The aims of the present work are to study the dynamical mechanisms responsible for onset of chaos in an SL connected to a resonator and to investigate how the frequency band of chaotic output depends on the voltage applied to the system. In particular, we revealed that appearance of chaos in such a system is accompanied by intermittent dynamics, whose scaling properties are typical for type I intermittency [35]. This type of intermittency is associated with the inverse tangent bifurcation, when two limit cycles (a stable and an unstable ones) merge and disappear [36]. Interestingly, such intermittent behavior is one of most generic fundamental mechanisms of transition to chaos [37-40] and to synchronization [41,42] in both purely deterministic and noisy dynamical systems of different origin. We demonstrate that in the system under study the relative spectral band width of generated chaotic voltage oscillations is very sensitive to applied voltage and can reach values more than $25 \%$. The results, which we report here, will be useful, e.g., for development and design of high-frequency chaos generators that use SL devices as key elements $[27,43]$.

In Sec. II we introduce a mathematical model which describes charge transport in an SL connected to a single-mode resonator. Section III studies the bifurcation mechanism of the onset of chaos. Section IV analyzes the evolution of spatiotemporal patterns of charge dynamics in SL and the band width of the corresponding high-frequency output. Finally, in Sec. V we summarize results and draw conclusions.

\section{MATHEMATICAL MODEL}

The device under study is schematically sketched in Fig. 1(a). In order to describe miniband transport along the SL axis (axis $x$ ) we consider a set of one-dimensional continuity and Poisson equations. In numerical simulations we use descretized version of these equations, following the approach suggested in Ref. [44]. Within the frames of this 

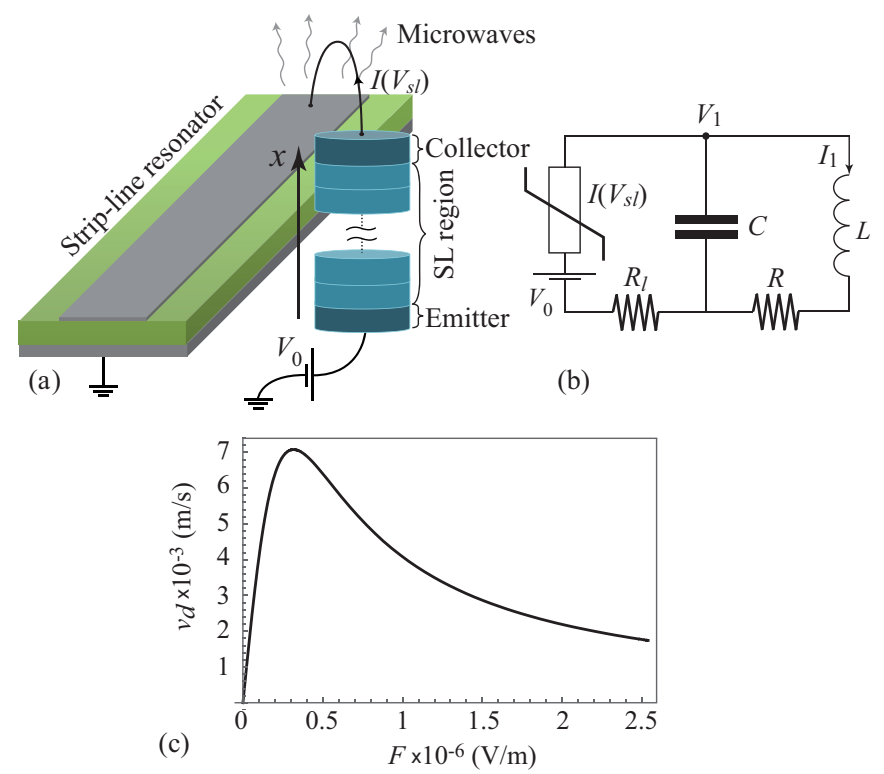

FIG. 1. (Color online) (a) Schematic diagram of a semiconductor superlattice coupled to an external strip-line resonator. (b) Equivalent circuit for an SL interacting with an external single-mode resonator. Here $C, L$, and $R$ are the equivalent capacitance, inductance, and resistance of the resonator, respectively, $I\left(V_{\mathrm{SL}}\right)$ is the current through the SL, with voltage $V_{\mathrm{SL}}$ dropped across it, and $V_{0}$ is the dc supply voltage. The load resistance is $R_{l}=0.1 \Omega$. (c) The dependence of the miniband drift velocity, $v_{d}(F)$, for the temperature $T=4.2 \mathrm{~K}$.

approach the transport region of SLs having length $L_{\mathrm{sl}}$ is split into a number of layers, each of width $\Delta x$. Then the time evolution of charge density $n_{m}(t)$ in the $m$ th layer is determined by the equation

$$
e \Delta x \frac{d n_{m}}{d t}=J_{m-1}-J_{m}, \quad m=1, \ldots, N,
$$

where $e$ is the electron charge, and $J_{m-1}$ and $J_{m}$ are the volume current densities on the left and right boundaries of the $m$ th layer. The latter can be calculated using the equation

$$
J_{m}=e n_{m} v_{d}\left(\bar{F}_{m}\right)
$$

where $\bar{F}_{m}$ is the mean field in the $m$ th layer $[3,44]$. It is assumed that charge transport is realized within the lowest miniband, when interminiband tunneling can be neglected. In this case the miniband drift velocity $v_{d}$ for the finite temperature $T$ and the given $\bar{F}_{m}$ can be calculated using the Esaki-Tsu-Romanov formalism [4]:

$$
v_{d}(\bar{F})=\frac{\Delta d}{2 \hbar} \frac{I_{1}\left(\Delta / 2 k_{B} T\right)}{I_{0}\left(\Delta / 2 k_{B} T\right)} \frac{e \bar{F} d \tau / \hbar}{1+(e \bar{F} d \tau / \hbar)^{2}},
$$

where $d=8.3 \mathrm{~nm}$ is the period of the SL, $\Delta=19.1 \mathrm{meV}$ is the miniband width, and $\tau$ is an effective scattering time, which takes into account both elastic and inelastic scattering events $[3,45,46]$. Parameter $k_{B}$ represents the Boltzmann constant and $I_{n}(x)$, where $n=0,1$, is a modified Bessel function of the first kind. In our calculations we fix $T=4.2 \mathrm{~K}$, and $\tau=176 \mathrm{fs}$, whose value we chose close to one from recent experiments [20,46,47]. The dependence of the electron drift velocity $v_{d}$ on electric field strength $F$ is illustrated in Fig. 1(c).
The function $v_{d}(F)$ demonstrates a characteristic maximum, which is associated with the onset of Bloch oscillations. With further growth of $F$ the effect of Bloch oscillations on electron dynamics becomes stronger. This leads to localization of electrons and the decrease of $v_{d}$. Although Eq. (3) was obtained for a static electric field $F$, it can be also used for a slowly oscillating electric field, when the miniband electrons can follow the ac field adiabatically [48-50], i.e., for $2 \pi f \tau \ll 1$, where $f$ is the frequency of an electric field applied. We note that for the value of $\tau$ used in our model this adiabatic limit spans up to several hundred $\mathrm{GHz}$.

The electric field $F_{m}$ at the left-hand edge of $m$ th layer is determined by the discretized Poisson equation

$$
F_{m+1}=\frac{e \Delta x}{\varepsilon_{0} \varepsilon_{r}}\left(n_{m}-n_{D}\right)+F_{m}, \quad m=1, \ldots, N .
$$

Here $\varepsilon_{0}$ and $\varepsilon_{r}=12.5$ are the absolute and relative permittivities, respectively, and $n_{D}=3 \times 10^{22} \mathrm{~m}^{-3}$ is the $n$-type doping density in the SL layers [46]. In the emitter and collector Ohmic contacts, $F=F_{0}$. Similarly to Refs. [20,44], we chose $N=480$ and $\Delta x=0.24 \mathrm{~nm}$, which is small enough to approximate a continuum and provide converge of a numerical solution.

Ohmic boundary conditions determine the current, $J_{0}=$ $\sigma F_{0}$, in the heavily doped emitter of electrical conductivity $\sigma=3788 \mathrm{Sm}^{-1}$ [46]. The voltage, $V_{\mathrm{sl}}$, applied to the device is a global constraint given by

$$
V_{\mathrm{sl}}=U+\frac{\Delta x}{2} \sum_{m=1}^{N}\left(F_{m}+F_{m+1}\right),
$$

where the voltage, $U$, dropped across the contacts includes the effect of charge accumulation and depletion in the emitter and collector regions and a contact resistance $R=17 \Omega[51,52]$. We calculate the current through SL as

$$
I(t)=\frac{A}{N+1} \sum_{m=0}^{N} J_{m}
$$

where $A=5 \times 10^{-10} \mathrm{~m}^{2}$ is the cross-sectional area of the SL $[3,44,46]$. We assume that the current $I(t)$ excites only one mode in the external resonator. In this case the resonator can be substituted by an equivalent lumped LRC circuit, which is characterized by frequency, $f_{Q}$, and by quality factor, $Q$. Such a single-mode approximation, which allows us to significantly simplify analysis and simulation of extended electrodynamic systems, is often used in a wide spectrum of problems, examples of which include helix structures in TWT amplifiers [53,54], klystrons [55], and Gunn diodes coupled to an external resonator [56,57].

The current $I(t)$ generated by the SL depends on voltage $V_{\text {sl }}$ applied to the device. This voltage includes a dc bias $V_{0}$ and ac voltage $V_{1}$ induced by the resonator, which, according to Fig. 1(b), can be described by the following equations:

$$
\begin{gathered}
C \frac{d V_{1}}{d t}=I\left(V_{\mathrm{sl}}\right)-I_{1}, \\
L \frac{d I_{1}}{d t}=-R I_{1}+V_{1}+R_{l} I\left(V_{\mathrm{sl}}\right) .
\end{gathered}
$$


Here $V_{\mathrm{sl}}=V_{0}-V_{1}, C, L$, and $R$ are the equivalent capacitance, inductance, and resistance of the resonator, respectively, and $R_{l}$ is the load resistance.

Equations (7) and (8) yield the resonance frequency $f_{Q}=$ $1 /(2 \pi \sqrt{L C})$ and the quality factor $Q=(1 / R) \sqrt{L / C}$. In our calculations we use the circuit parameters from recent experiment [20]: $R_{l}=0.1 \Omega, R=0.5 \Omega, C=3.0 \mathrm{pF}$, and $L=0.17 \mathrm{nH}$

\section{TRANSITION TO CHAOS}

Depending on the bias voltage $V_{0}$, the model (1)-(8) demonstrates either constant or oscillating electric current $I(t)$. In a single SL without external resonator, the periodic current oscillations appear when $V_{0}$ exceeds a certain threshold value $\approx 0.34 \mathrm{~V}$. These oscillations remain periodic for any larger values of $V_{0}$. However, connecting the SL to a resonator can cause additional instabilities eventually leading to generation of chaos [20].

Figure 2 presents a bifurcation diagram illustrating different dynamical regimes of an SL coupled to a resonator for a range of bias voltage $V_{0}=400-900 \mathrm{mV}$ (a) and the dependence of the frequency of the current $I(t)$ generated by the SL without a resonator on $V_{0}$ (b). The plot in Fig. 2(a) shows local maxima of voltage oscillations $V_{1}(t)$ calculated for different values of $V_{0}$. For low bias voltage $V_{0}=400-500 \mathrm{mV}$ the diagram reveals periodic oscillations, which for each value of $V_{0}$ are represented by one or a few points. The range of $V_{0}=500$ $550 \mathrm{mV}$ demonstrates two regions of irregular dynamics of $V_{1}(t)$ (erratic sets of points) split by a region of period-three oscillations. Further increase of $V_{0}$ regularizes the oscillations; however, for high enough voltage $V_{0}>840 \mathrm{mV}$, the diagram again displays a large area of chaotic dynamics. Notably, as is seen from Fig. 2(b), chaos in the system emerges when the frequency of resonator $\left(f_{Q}=13.81 \mathrm{GHz}\right)$ is detuned from the frequency of $I(t)$.
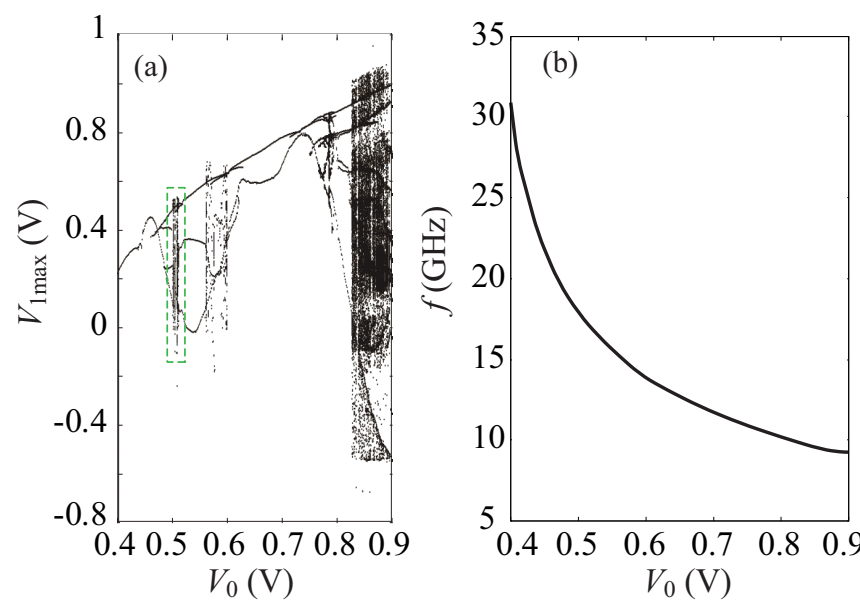

FIG. 2. (Color online) (a) Bifurcation diagram illustrating the evolution of voltage oscillations in resonator $V_{1}$ with variation of the supply voltage $V_{0}$ for $f_{Q}=13.81 \mathrm{GHz}$ and $Q=150$; (b) dependence of the frequency of $I(t)$, generated by the SL without resonator, upon $V_{0}$.
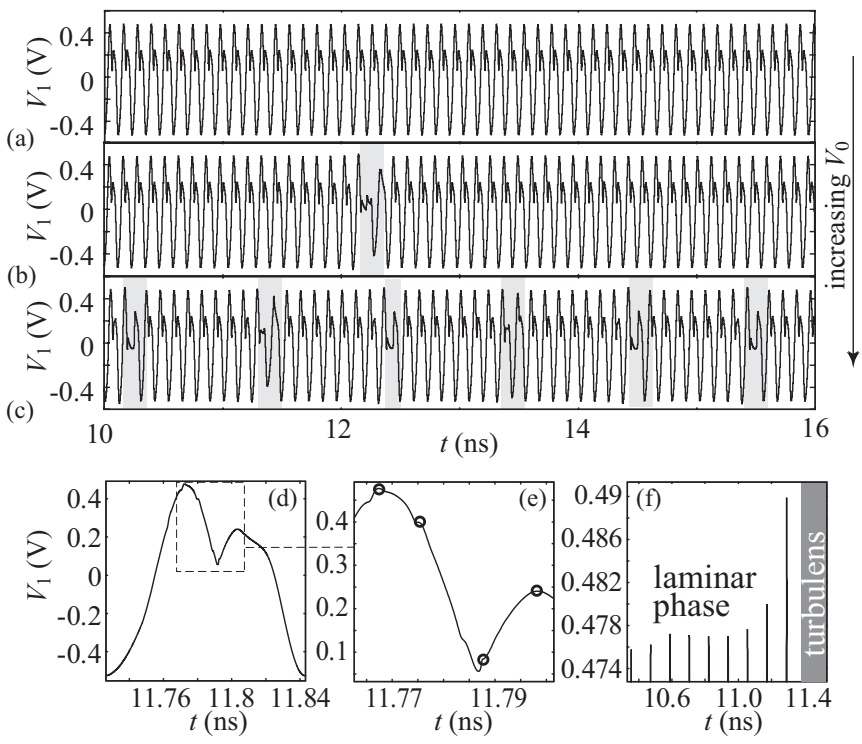

FIG. 3. Time series of $V_{1}(t)$ for (a) $V_{0}=510.68 \mathrm{mV}$, (b) $V_{0}=$ $510.7 \mathrm{mV}$, (c) $V_{0}=510.75 \mathrm{mV}$; turbulent phases in (b) and (c) are marked by a gray stripe. (d) Single period of voltage oscillations for $V_{0}=510.7 \mathrm{mV}$ and (e) its zoomed part. The circles in (e) show four local maxima. (f) Change of the amplitude of $V_{1}(t)$ within the laminar phase; the gray box indicates the turbulent phase. External resonator is characterized by $f_{Q}=13.81 \mathrm{GHz}$ and $Q=150$.

In order to better illustrate the transition to chaos in the region framed by the dashed rectangle in Fig. 2(a), we calculate time realizations of $V_{1}(t)$ for three characteristic values of $V_{0}$ [see Figs. 3(a)-3(c)]. As Fig. 3(a) shows, for $V_{0}=510.68 \mathrm{mV}$ the voltage $V_{1}$ demonstrates pure periodic behavior. For $V_{0}=510.7 \mathrm{mV}$ [Fig. 3(b)] the long periodic phases of $V_{1}(t)$ are occasionally intermitted by turbulent oscillations, which are marked in Fig. 3(b) by a gray strip. It is also seen that the chaotic behavior arises on the basis of a period-four cycle. On order to illustrate this, one period of this cycle is shown in Figs. 3(d) and 3(e), where four local maxima of $I(t)$ are indicated by circles.

Further raising of $V_{0}$ up to $510.75 \mathrm{mV}$ leads to rapid increase of frequency of the turbulent events [Fig. 3(c)]. Such evolution of the time realizations $V_{1}(t)$ with variation of $V_{0}$ suggests the transition of chaos via the intermittency phenomenon [35]. Remarkably, the height of local maxima is increasing from the start of the laminar region to its end [Fig. 3(f)], which is quite typical for the type I intermittency [37].

To get deeper insight into observed intermittent dynamics, we investigate how the mean length of laminar phase $\langle\tau\rangle$ changes with increase of voltage $V_{0}$. Figure 4 presents the dependence of $\langle\tau\rangle$ upon the supercriticality parameter $V_{0}-V_{\text {0crit }}$, where $V_{\text {ocrit }}$ is the value of the critical voltage $V_{0}$ corresponding to the onset of the intermittent dynamics. For the chosen set of the parameters we estimate $V_{0 \text { crit }}=510.69 \mathrm{mV}$. In our calculations shown in Fig. 4 we analyze $V_{1}(t)$ within the time intervals, which are long enough to provide us with at least $10^{3}$ turbulent phases for $V_{0}-V_{0 \text { crit }} \in[2,10] \mu \mathrm{V}$. One can see that the calculated dependence of $\langle\tau\rangle$ upon $V_{0}-V_{0 \text { crit }}$ (black circles) in Fig. 4 is close to the power law with exponent 


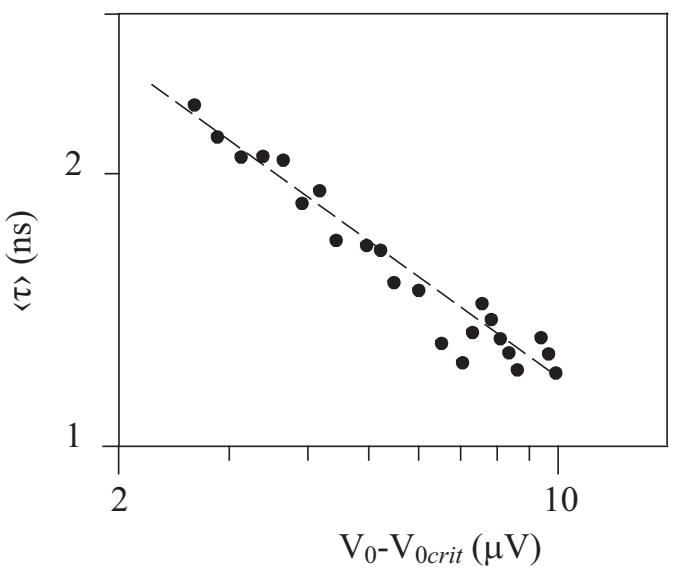

FIG. 4. The log-log plot of the mean laminar phase length $\langle\tau\rangle$ versus criticality parameter $\left(V_{0}-V_{0 \text { crit }}\right)$ (points) and its approximation $\langle\tau\rangle \sim\left(V_{0}-V_{0 \text { crit }}\right)^{-0.5}$ (dashed line). Here $V_{\text {0crit }}=510.69 \mathrm{mV}$; parameters of external resonator: frequency $f_{Q}=13.81 \mathrm{GHz}$, quality factor $Q=150$.

-0.5 (dashed line):

$$
\langle\tau\rangle=\alpha\left(V_{0}-V_{0 \text { crit }}\right)^{-0.5},
$$

which confirms the development of type I intermittency $[36,37]$.

It is known that the transition to chaos via a type I intermittency involves the inverse tangent bifurcation, in which two limit cycles (a stable and an unstable one) merge and disappear. This instability leads to formation of a specific pattern in Poincare map, which is associated with emergence of a "channel" between the map points and the diagonal [36]. Figure 5(a) demonstrates the Poincaré map of $V_{1}(t)$, when $V_{0}=510.71 \mathrm{mV}$ slightly exceeds $V_{0 \text { crit. }}$. It was constructed numerically as dependence of $V_{1(n+1)}$ upon $V_{1(n)}$, where $V_{1(n)}$ is the $n$th local maximum in a time realization of $V_{1}(t)$. Since we deal with period-four limit cycles, every fourth local maxima was taken for this map. As one can see, the map indeed demonstrates a narrow channel formed due to an inverse tangent bifurcation, which becomes more evident in a zoomed fragment of the map presented in Fig. 5(b). Wandering of the
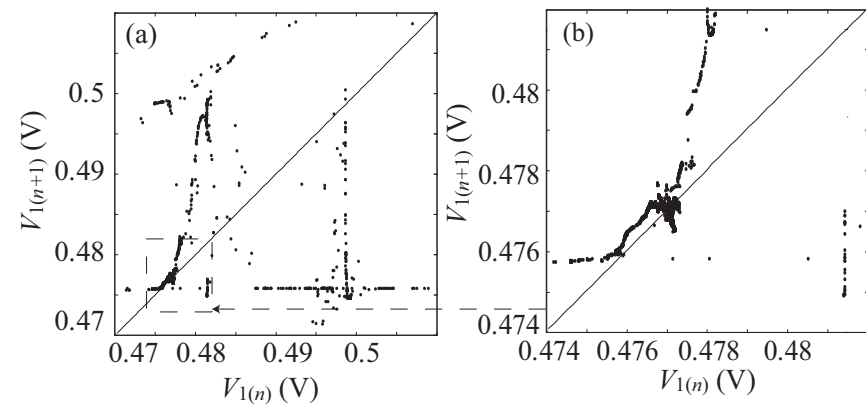

FIG. 5. (a) Poincaré map of voltage oscillations in a resonator when $V_{0}$ exceeds the critical value. Bias voltage $V_{0}=510.71 \mathrm{mV}$. Parameters of external resonator: frequency $f_{Q}=13.81 \mathrm{GHz}$, quality factor $Q=150$. (b) Zoomed region of the Poincaré map containing a narrow channel presence which leads to the long laminar phase. In both figures the solid line corresponds to the diagonal.

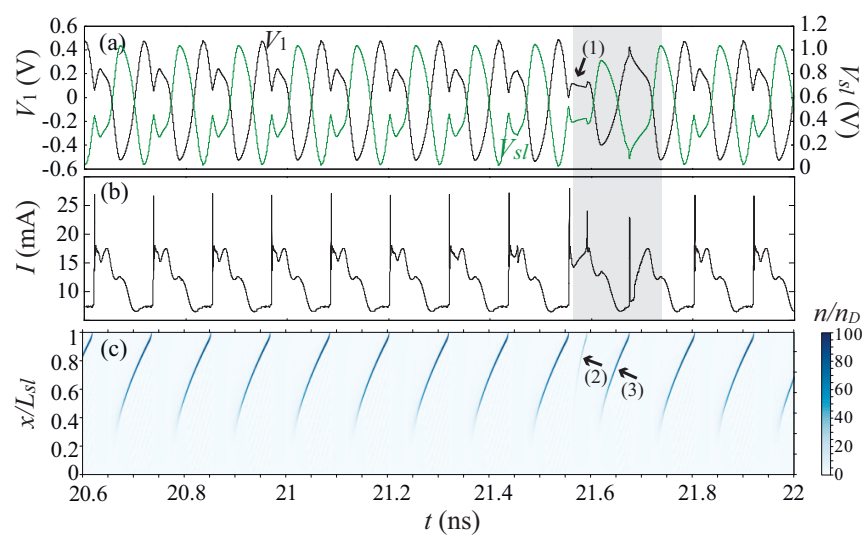

FIG. 6. (Color online) Time series of (a) $V_{1}(t)$ (back) and $V_{\mathrm{s}}$ (gray/green online), (b) $I(t)$, and (c) spatiotemporal distributions of charge in SL $n(x, t)$ for $V_{0}=510.75 \mathrm{mV}, f_{Q}=13.81 \mathrm{GHz}$, and $Q=150$. The turbulent phase in the time series is indicated by gray areas.

map points within this channel corresponds to the laminar phase of oscillations $V_{1}(t)$, whereas leaving the channel leads to appearance of turbulent dynamics. Specifically in Fig 5, the turbulent phase of oscillations is represented by a complex structure of the map for $V_{1}>480 \mathrm{mV}$. After spending some time in this region, the phase trajectory returns to the vicinity of $V_{1} \approx 477 \mathrm{mV}$, where relaminarization of oscillations is realized.

To understand the physical processes generating the turbulent phase we analyze a time realization of $V_{1}(t)$ and $V_{\mathrm{sl}}(t)$ [Fig. 6(a)], $I(t)$ [Fig. 6(b)] together with the corresponding spatiotemporal pattern of $n(t, x)$ [Fig. 6(c)]. The charge density $n(t, x)$ is presented by a color scale in units of the doping density $n_{D}$. Figure 6 reveals that the laminar phases of $V_{1}(t)$ are characterized by regular behavior of high-density charge domains traveling along the SL. Each domain is generated when the values of $V_{1}$ achieve a minimum. This time moment corresponds to the maximal value of $V_{\mathrm{sl}}=V_{0}-V_{1}$, which triggers domain formation, and while a domain propagates along the SL, no new domains can be generated. As we mentioned above, the decoupled SL and the resonator have different characteristic time scales. However, for small $V_{0}$, the interaction of the resonator and the SL coordinates oscillations of $I(t)$ and the resonator response $V_{1}(t)$. Growth of $V_{0}$ leads to increase of the amplitude of $I(t)$, which excites the resonator. Therefore, the response of the resonator $V_{1}(t)$ becomes more powerful. Occasionally, interaction of the SL and the resonator produces a phase slip between $I(t)$ and $V_{1}(t)$, which leads to local decrease of $V_{1}$ [arrow (1) in Fig. 6(a)] and thus growth of $V_{\mathrm{sl}}$. Eventually, raising $V_{\mathrm{sl}}$ exceeds the threshold value and launches an additional domain [arrow (2) in Fig. 6(c)], which imposes disorder in the queue of domains. This causes further perturbations in $I(t)$ forming a turbulent phase in $V_{1}(t)$. A slight increase of $V_{0}$ makes the phase slips more often, which develops the chaos.

\section{BROADBAND GENERATION}

Next, we consider a wide region of chaos, which can be found in Fig. 2(a) for $V_{0}>820 \mathrm{mV}$. Our analysis showed 


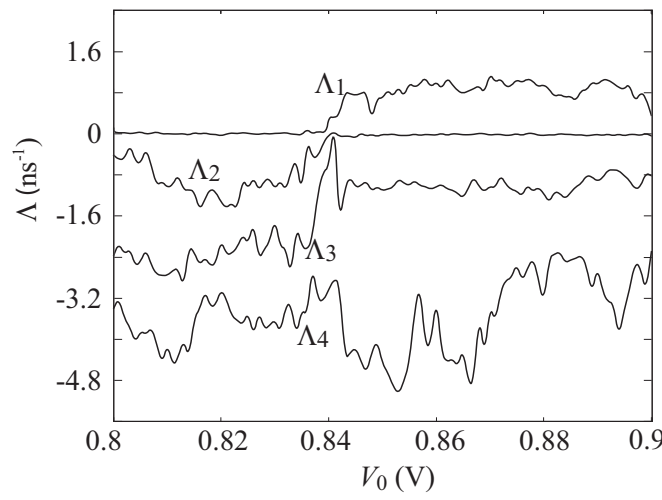

FIG. 7. Four largest Lyapunov exponents $\Lambda_{1}-\Lambda_{4}$ vs $V_{0}$ for $f_{Q}=$ $13.81 \mathrm{GHz}$ and $Q=150$.

that the transition to chaos in this range of $V_{0}$ is realized according to the same intermittent scenario as described above. However, this region of chaos is characterized by more powerful oscillations of $V_{1}(t)$ and has much less prominent periodic windows, which alternates with chaos with variation of $V_{0}$.

The evolution of the spectrum of the Lyapunov exponents corresponding to this transition is shown in Fig. 7. The figure demonstrates the dependence of four largest Lyapunov exponents $\Lambda_{1}-\Lambda_{4}$ upon $V_{0}$ calculated by method discussed in Refs. [58,59]. For $V_{0}<841 \mathrm{mV}$ the largest Lyapunov exponent is $\Lambda_{1}=0$, whereas $\Lambda_{2}, \Lambda_{3}, \Lambda_{4}<0$. Such a spectrum of the Lyapunov exponent evidences generation of regular periodic current and voltage oscillations in the system. With an increase of $V_{0}$, exponent $\Lambda_{1}$ becomes positive, $\Lambda_{2}$ grows to be zero, and $\Lambda_{3}$ together with $\Lambda_{4}$ remain negative. Appearance of a positive Lyapunov exponent corresponds to the onset of chaos. Remarkably, the positive Lyapunov exponent, which measures the level of unpredictability of the generated dynamics, varies only slightly within a considerable range of $V_{0} \in[850,900] \mathrm{mV}$.

Figure 8 presents typical time realizations of voltage $V_{1}(t)$ $[(\mathrm{a}),(\mathrm{d}),(\mathrm{g}),(\mathrm{j})]$, the current generated by SL $I(t)[(\mathrm{b}),(\mathrm{e})$, (h), (k)], and charge concentration $n(x, t)$ [(c), (f), (i), (l)] for different $V_{0}$ from the range of $V_{0}>820 \mathrm{mV}$. For lower voltage $V_{0}=830 \mathrm{mV} V_{1}(t)$ [Fig. 8(a)] demonstrates regular period-four oscillations, which are coordinated in time with oscillations of $I(t)$ [Fig. 8(b)]. Once a charge domain arrives to the SL collector [Fig. 8(c)], it creates a massive peak in the current $I(t)$ [Fig. 8(b)]. Each such current peak corresponds to two successive oscillations of $V_{1}(t)$ [Fig. 8(a)]. The regularity of these oscillations is confirmed by zero values of the largest Lyapunov exponent shown in Fig. 7. For larger value of $V_{0}=852 \mathrm{mV}$ the oscillations in the resonator $V_{1}(t)$ [Fig. 8(d)] start to demonstrate intermittent behavior, where regular and turbulent phases alternate. In this regime the motion of the charge domains becomes irregular [Fig. 8(f)], which generates quite erratic peaks in the current $I(t)$ [Fig. 8(e)]. As Fig. 7 shows, this dynamics is characterized by a positive largest Lyapunov exponent, which confirms the appearance of chaos in the system. Further increase of $V_{0}$ up to $858 \mathrm{mV}$ makes the generated chaos even more complex by producing a new phase of intermittent behavior associated with small amplitude
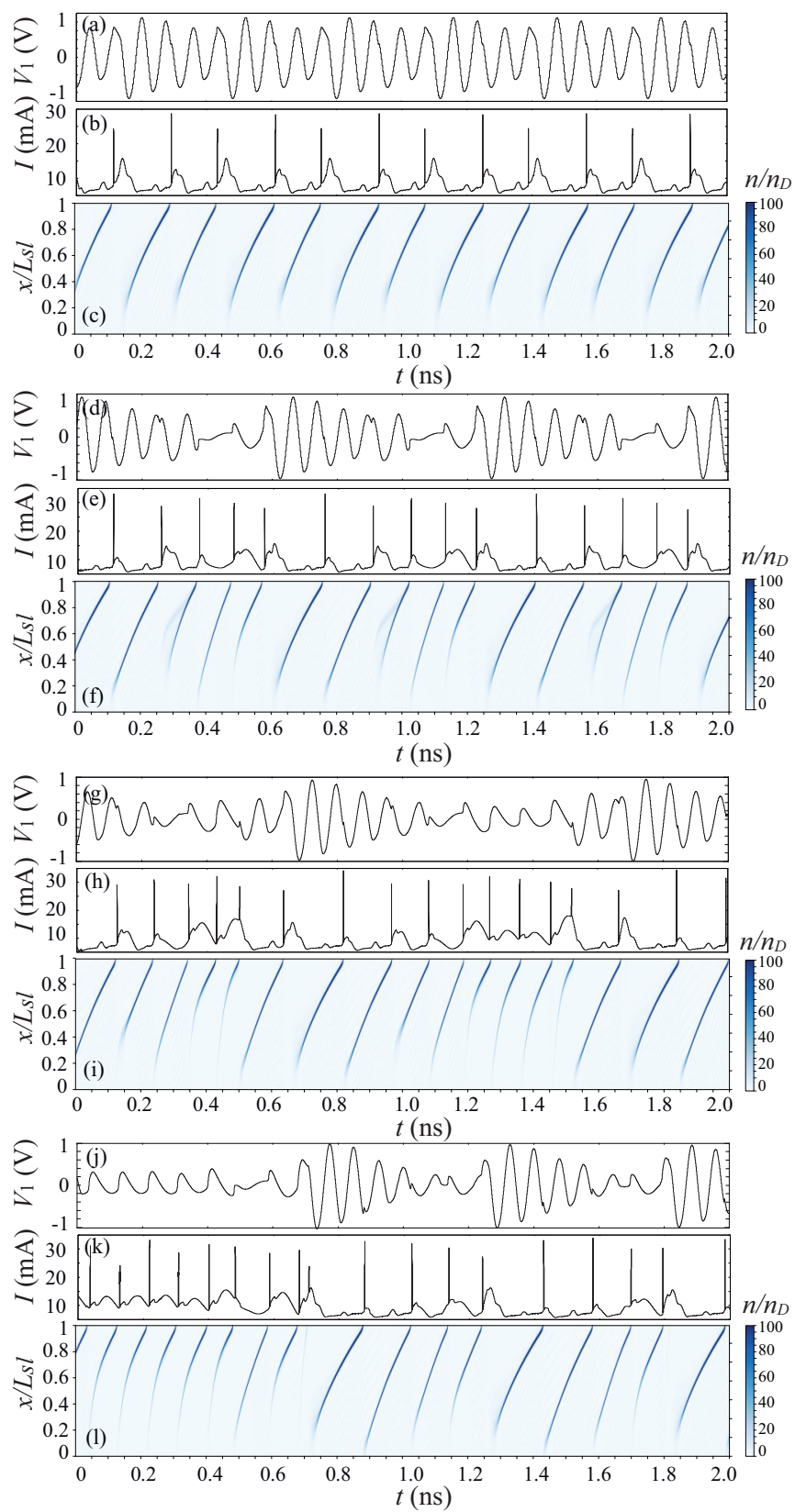

FIG. 8. (Color online) Time series of voltage (a), (d), (g), (j), and current (b), (e), (h), (k) oscillations, and spatiotemporal distributions of charge in SL illustrated domain transport (c), (f), (i), (l) for different bias voltage: (a), (b), (c) $830 \mathrm{mV}$, (d), (e), (f) $852 \mathrm{mV}$, (g), (h), (i) $858 \mathrm{mV}$, (j), (k), (l) $863 \mathrm{mV}$. Parameters of external resonator: frequency $f_{Q}=13.81 \mathrm{GHz}$, quality factor $Q=150$.

oscillations of $V_{1}(t)$ [see, e.g., the time interval 1.2-1.6 ns in Fig. 8(g)]. These oscillations correspond to generation of a series of fast-moving charge domains [Fig. 8(i)], which produce high-frequency current oscillations [Fig. 8(h)]. This phase of chaotic oscillations becomes even more prominent for higher voltage $V_{0}=863 \mathrm{mV}$, as one can see in Figs. 8(j)-8(1)] within the time interval $0-0.5 \mathrm{~ns}$.

In order to study the spectral characteristics of the generated signals, we calculate power spectral density $S(f)$ of the voltage oscillations in the resonator $V_{1}(t)$. Figures $9($ a) $-9(d)$ illustrate 

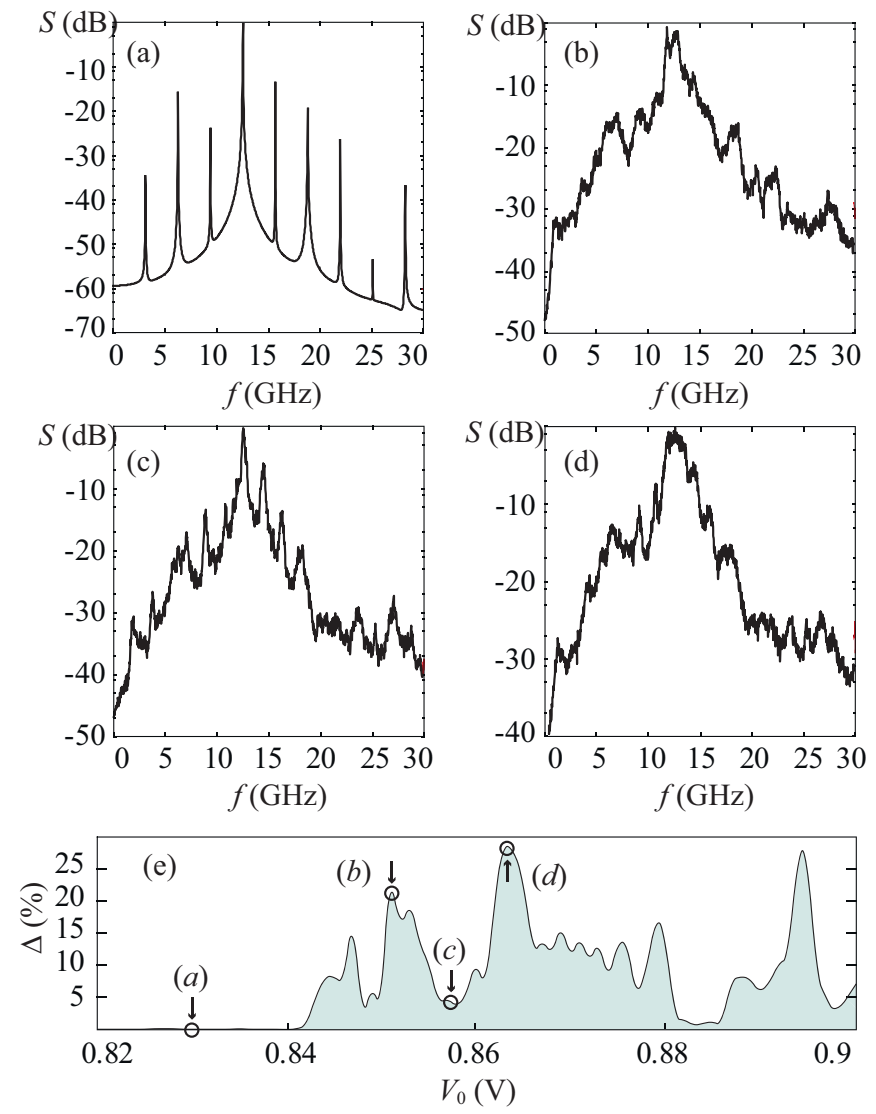

FIG. 9. (Color online) (a) Power spectral density of voltage oscillations before the transition to chaos for (a) $V_{0}=830 \mathrm{mV}$; and after transition to chaos for (b) $V_{0}=852 \mathrm{mV}$, (c) $V_{0}=858 \mathrm{mV}$, and (d) $V_{0}=863 \mathrm{mV}$. (e) Bandwidth vs the bias voltage $V_{0}$. Parameters of external resonator are $f_{Q}=13.81 \mathrm{GHz}$ and $Q=150$.

evolution of $S(f)$ with variation of the bias voltage $V_{0}$. For $V_{0}=830 \mathrm{mV}$ [Fig. 9(a)] the oscillations of $V_{1}$ have a discrete spectrum $S(f)$ with the dominant peak at $f \approx 12.72 \mathrm{GHz}$ corresponding to the basic frequency of oscillations. The peak is surrounded by harmonics and subharmonics delimited by frequency interval $\Delta f \approx 3.18 \mathrm{GHz}$. This structure of the spectral power density reflects the period-four regular oscillations, presented in Fig. 8(a). For larger voltages $V_{0}=$ $852 \mathrm{mV}$ [Fig. 9(b)], $V_{0}=858 \mathrm{mV}$ [Fig. 9(c)], and $V_{0}=$ $863 \mathrm{mV}$ [Fig. 9(d)], the position of the dominant peak in the spectra remains almost the same. However, these spectra become broadband and continuous, confirming generation of developed chaos for larger values of $V_{0}$. Notably, spectra of chaotic oscillations $S(f)$ presented in Figs. 9(b)-9(d) have quite a similar shape. We quantitatively characterize the bandwidth of the generated signal using the conventional "-3 dB" definition [60-62]. In this case the bandwidth $\Delta_{f}$ is defined as the difference $\Delta_{f}=f_{b 2}-f_{b 1}$ between the upper $\left(f_{b 2}\right)$ and lower $\left(f_{b 1}\right)$ boundary of the frequency range, within which the power density exceeds half of the maximum spectral density, which is observed for the frequency $f_{m}$.

The relative bandwidth is then calculated as $\Delta=\Delta_{f} / f_{m} \times$ $100 \%$. The dependence of $\Delta\left(V_{0}\right)$ for $V_{0}>820 \mathrm{mV}, f_{Q}=$ 13.81, and $Q=150$ is presented in Fig. 9(e). Arrows (a)-(d) point the values of $V_{0}$ corresponding to the Figs. 9(a)-9(d), respectively. For $V_{0}$ between 0.820 and $0.841 \mathrm{mV}$ the relative bandwidth of $V_{1}$ is practically zero reflecting the periodicity of $V_{1}(t)$ oscillations. However, when $V_{0}$ exceeds $0.841 \mathrm{mV}$ the bandwidth of $V_{1}(t)$ becomes finite, implicating the onset of chaos. Interestingly, despite the close values of the largest Lyapunov exponents [see Fig. 7] for chaos, whose power spectral density are shown in Figs. 9(b)-9(d), the bandwidth of the corresponding oscillations changes significantly between $\sim 650 \mathrm{MHz}(\Delta \approx 4.2 \%)$ for $V_{0}=858 \mathrm{mV}$ [Fig. 9(c)] and $\sim 3 \mathrm{GHz}(\Delta \approx 28.1 \%)$ for $V_{0}=863 \mathrm{mV}$ [Fig. 9(d)]. This fact proposes that $V_{0}$ can be used for effectively controlling the bandwidth of the signal generated in the SL coupled to a resonator.

\section{CONCLUSION}

In conclusion, we demonstrated theoretically that an SL coupled to a resonator can generate chaotic high-frequency oscillations of current and voltage. This chaos is developed by following the intermittency scenario, which is characterized by alternating laminar and turbulent phases of the oscillations. With change of the bias voltage $V_{0}$ the turbulent phases occur more often, thus developing chaos in the generated oscillations. Appearance of chaos was confirmed by calculation of spectra and the Laypunov exponents. The analysis of the laminar phase length for different bias voltage and of the topology of Poincaré maps revealed that chaos is developed via type I intermittency [35]. We found out that intermittency is generated when the resonant frequency of the resonator is detuned from the frequency of the current oscillations generated by an isolated SL. In this case, for certain ranges of $V_{0}$ response oscillations in the resonator become uncoordinated with the driving current oscillations generated by the SL. This leads to formation of the turbulent phase and development of chaos in the system.

Our results suggest that SLs coupled to a single-mode resonator can be used as a tunable broadband generator, whose relative bandwidth can be controlled by voltage within a significant range 0\%-28\%, which for the SL model we used corresponds to $0-3.6 \mathrm{GHz}$. These characteristics match or even exceed similar parameters of other solid-state and vacuum sources of chaotic signals [25,63-68]. The latter makes the SL coupled to a resonator promising for using it as a key element in broadband sources of chaotic microwaves or fast random number generators $[20,27,43,69]$, which could have applications in high-speed chaotic communications and spreadspectrum spectroscopy. In addition, previous works [24,28] have shown theoretically and experimentally that noise can significantly enhance the chaos generated in weakly coupled SLs. Taking into account these results we can assume that the inclusion of the noise in our model should lead to more developed chaos in charge dynamics in the SL under study and, thus, to the increase of bandwidth of microwave output. We believe that our findings can also be relevant to other devices, demonstrating space charge dynamics, e.g., Gunn diodes [70,71], and vacuum microwave devices with a virtual cathode $[72,73]$. However, this requires further investigations.

In obtaining the presented results we made at least three significant simplifications, namely, we neglected intermini- 
band tunneling, assumed adiabatic change of $v_{d}$ for a timedependent electric field applied, and substituted a resonator with an lumped equivalent circuit. Although all are reasonable approximations, and can be valid for a certain class of devices [20,46,47], from the viewpoint of applications it would be useful to study more general cases, when one or more simplifications made are invalid. It is especially interesting to consider the case of a generic resonator, which cannot be described in terms of equivalent lumped parameters. Such resonators allow modes of complicated shapes or even multimode competition. All those factors cold dramatically affect spectral content of the generated signal and thus its bandwidth.

\section{ACKNOWLEDGMENTS}

This work has been supported by the Russian Science Foundation (Grant No. 14-12-00222). We are thankful to Dr. Kirill Alekseev for useful discussions and valuable comments related to this paper.
[1] L. Esaki and R. Tsu, IBM J. Res. Develop. 14, 61 (1970).

[2] A. Y. Shik, Sov. Phys. Semicond. 8, 1195 (1975).

[3] A. Wacker, Phys. Rep. 357, 1 (2002).

[4] Y. A. Romanov, Optika i Spektroskopiya 33, 917 (1972).

[5] C. P. Endres, F. Lewen, T. F. Giesen, S. Schlemmer, D. G. Paveliev, Y. I. Koschurinov, V. M. Ustinov, and A. E. Zhucov, Rev. Sci. Instrum. 78, 043106 (2007).

[6] E. Schomburg, T. Blomeier, K. Hofbeck, J. Grenzer, S. Brandl, I. Lingott, A. A. Ignatov, K. F. Renk, D. G. Pavel'ev, Yu. Koschurinov, B. Ya. Melzer, V. M. Ustinov, S. V. Ivanov, A. Zhukov, and P. S. Kop'ev, Phys. Rev. B 58, 4035 (1998).

[7] T. M. Fromhold, A. A. Krokhin, C. R. Tench, S. Bujkiewicz, P. B. Wilkinson, F. W. Sheard, and L. Eaves, Phys. Rev. Lett. 87 , 046803 (2001).

[8] T. Hyart, N. V. Alexeeva, J. Mattas, and K. N. Alekseev, Phys. Rev. Lett. 102, 140405 (2009).

[9] E. E. Mendez, F. Agulló-Rueda, and J. M. Hong, Phys. Rev. Lett. 60, 2426 (1988).

[10] T. Hyart, A. V. Shorokhov, and K. N. Alekseev, Phys. Rev. Lett. 98, 220404 (2007).

[11] V. V. Makarov, A. E. Hramov, A. A. Koronovskii, K. N. Alekseev, V. A. Maksimenko, M. T. Greenaway, T. M. Fromhold, O. I. Moskalenko, and A. G. Balanov, Appl. Phys. Lett. 106, 043503 (2015)

[12] F. Klappenberger, A. A. Ignatov, S. Winnerl, E. Schomburg, W. Wegscheider, K. F. Renk, and M. Bichler, Appl. Phys. Lett. 78, 1673 (2001).

[13] J. F. O’Hara, J. M. O. Zide, A. C. Gossard, A. J. Taylor, and R. D. Averitt, Appl. Phys. Lett. 88, 251119 (2006).

[14] A. A. Ignatov and A.-P. Jauho, J. App. Phys. 85, 3643 (1999).

[15] V. A. Maksimenko, V. V. Makarov, A. A. Koronovskii, K. N. Alekseev, A. G. Balanov, and A. E. Hramov, Europhys. Lett. 109, 47007 (2015)

[16] G. S. Nusinovich, A. N. Vlasov, and T. M. Antonsen, Phys. Rev. Lett. 87, 218301 (2001).

[17] V. B. Taranenko, I. Ganne, R. J. Kuszelewicz, and C. O. Weiss, Phys. Rev. A 61, 063818 (2000).

[18] V. Grigoriev and F. Biancalana, Phys. Rev. A 83, 043816 (2011).

[19] J. Petráček, Y. Ekşioğlu, and A. Sterkhova, Opt. Commun. 318, 147 (2014).

[20] A. E. Hramov, V. V. Makarov, A. A. Koronovskii, S. A. Kurkin, M. B. Gaifullin, N. V. Alexeeva, K. N. Alekseev, M. T. Greenaway, T. M. Fromhold, A. Patané, F. V. Kusmartsev, V. A. Maksimenko, O. I. Moskalenko, and A. G. Balanov, Phys. Rev. Lett. 112, 116603 (2014).

[21] J. C. Cao and X. L. Lei, Phys. Rev. B 60, 1871 (1999).
[22] A. Amann, J. Schlesner, A. Wacker, and E. Schöll, Phys. Rev. B 65, 193313 (2002)

[23] A. G. Balanov, D. Fowler, A. Patanè, L. Eaves, and T. M. Fromhold, Phys. Rev. E 77, 026209 (2008).

[24] M. Alvaro, M. Carretero, and L. L. Bonilla, Europhys. Lett. 107, 37002 (2014).

[25] Y. Zhang, J. Kastrup, R. Klann, K. H. Ploog, and H. T. Grahn, Phys. Rev. Lett. 77, 3001 (1996).

[26] Y. Y. Huang, W. Li, W. Ma, H. Qin, and Y. Zhang, Chin. Sci. Bull. 57, 2070 (2012).

[27] W. Li, I. Reidler, Y. Aviad, Y. Huang, H. Song, Y. Zhang, M. Rosenbluh, and I. Kanter, Phys. Rev. Lett. 111, 044102 (2013).

[28] Yu. Bomze, R. Hey, H. T. Grahn, and S. W. Teitsworth, Phys. Rev. Lett. 109, 026801 (2012).

[29] A. Uchida, K. Amano, M. Inoue, K. Hirano, S. Naito, H. Someya, I. Oowada, T. Kurashige, M. Shiki, S. Yoshimori, K. Yoshimura, and P. Davis, Nature Photonics 2, 728 (2008).

[30] A. A. Koronovskii, O. I. Moskalenko, and A. E. Hramov, Physics-Uspekhi 52, 1213 (2009).

[31] D. Materassi and M. Basso, Intl. J. Bifurcat. Chaos 18, 567 (2008).

[32] O. I. Moskalenko, A. A. Koronovskii, and A. E. Hramov, Phys. Lett. A 374, 2925 (2010).

[33] B. Schneier, Applied Cryptography (Wiley, New York, 1996).

[34] J. G. Wu, X. Tang, Z. M. Wu, G. Q. Xia, and G. Y. Feng, Laser Phys. 22, 1476 (2012).

[35] Y. Pomeau and P. Manneville, Comm. Math. Phys. 74, 189 (1980).

[36] P. Berge, Y. Pomeau, and C. Vidal, L'Ordre Dans Le Chaos (Hermann, Paris, 1988).

[37] H. G. Schuster and W. Just, Deterministic Chaos: An Introduction (Wiley-VCH, Weinheim, 2005).

[38] E. F. Manffra, I. L. Caldas, R. L. Viana, and H. J. Kalinowski, Nonlinear Dynam. 27, 185 (2002).

[39] L. Russo, P. Altimari, E. Mancusi, P. L. Maffettone, and S. Crescitelli, Chaos, Solitons Fractals 28, 682 (2006).

[40] K. Gustavo, E. Sergio, and R. Ezequiel, Nonlinear Dynam. 77, 455 (2014).

[41] A. E. Hramov, A. A. Koronovskii, M. K. Kurovskaya, A. A. Ovchinnikov, and S. Boccaletti, Phys. Rev. E 76, 026206 (2007).

[42] A. E. Hramov, A. A. Koronovskii, M. K. Kurovskaya, and O. I. Moskalenko, Phys. Lett. A 375, 1646 (2011).

[43] H.-P. Ren, M. S. Baptista, and C. Grebogi, Phys. Rev. Lett. 110, 184101 (2013).

[44] M. T. Greenaway, A. G. Balanov, E. Schöll, and T. M. Fromhold, Phys. Rev. B 80, 205318 (2009). 
[45] E. Schomburg, S. Brandl, S. Winnerl, K. F. Renk, N. N Ledentsov, V. M. Ustinov, A. Zhukov, P. S. Kop'ev, H.-W. Hübers, J. Schubert, H. P. Röser, A. Rosenauer, D. Litvinov, D. Gerthsen, and J. M. Chamberlain, Physica E 7, 814 (2000).

[46] T. M. Fromhold, A. Patanè, S. Bujkiewicz, P. B. Wilkinson, D. Fowler, D. Sherwood, S. P. Stapleton, A. A. Krokhin, L. Eaves, M. Henini, N. S. Sankeshwar, and F. W. Sheard, Nature (London) 428, 726 (2004).

[47] N. Alexeeva, M. T. Greenaway, A. G. Balanov, O. Makarovsky, A. Patanè, M. B. Gaifullin, F. Kusmartsev, and T. M. Fromhold, Phys. Rev. Lett. 109, 024102 (2012).

[48] E. Schomburg, A. A. Ignatov, J. Grenzer, K. F. Renk, D. G. Pavel'ev, Yu. Koschurinov, Ja. B. Melzer, S. Ivanov, S. Schaposchnikov, and P. S. Kop'ev, Appl. Phys. Lett. 68, 1096 (1996).

[49] S. Winnerl, E. Schomburg, J. Grenzer, H.-J. Regl, A. A. Ignatov, A. D. Semenov, K. F. Renk, D. G. Pavel'ev, Yu. Koschurinov, B. Melzer, V. Ustinov, S. Ivanov, S. Schaposchnikov, and P. S. Kop'ev, Phys. Rev. B 56, 10303 (1997).

[50] F. Klappenberger, K. N. Alekseev, K. F. Renk, R. Scheuerer, E. Schomburg, S. J. Allen, G. R. Ramian, J. S. S. Scott, A. Kovsh, V. Ustinov, and A. Zhukov, Eur. Phys. J. B 39, 483 (2004).

[51] M. T. Greenaway, A. G. Balanov, and T. M. Fromhold, in Nonlinear Laser Dynamics. From Quantum Dots to Cryptography, edited by K. Lüdge (Wiley-VCH, Weinheim, 2012), p. 111.

[52] A. G. Balanov, M. T. Greenaway, A. A. Koronovskii, O. I. Moskalenko, A. O. Sel'skii, T. M. Fromhold, and A. E. Hramov, J. Exp. Theor. Phys. 114, 836 (2012).

[53] W. Qiu, H. J. Lee, J. P. Verboncoeur, and C. K. Birdsall, IEEE Trans. Plasma Sci. 29, 911 (2001).

[54] I. J. Morey and C. K. Birdsall, IEEE Trans. Plasma Sci. 18, 482 (1990).

[55] T. Shintake, Nucl. Instrum. Method Phys. Res. A363, 83 (1995).

[56] K. Chang and L.-H. Hsieh, Microwave Ring Circuits and Related Structures (Wiley, New York, 2004).
[57] S. Garcia, I. Iniguez-de-la-Torre, S. Perez, J. Mateos, and T. Gonzalez, J. Appl. Phys. 114, 074503 (2013).

[58] A. E. Hramov, A. A. Koronovskii, V. A. Maksimenko, and O. I. Moskalenko, Phys. Plasmas 19, 082302 (2012).

[59] A. A. Koronovskii, A. E. Hramov, V. A. Maximenko, O. I. Moskalenko, K. N. Alekseev, M. T. Greenaway, T. M. Fromhold, and A. G. Balanov, Phys. Rev. B 88, 165304 (2013).

[60] J. D. Taylor, editor, Ultrawideband Radar: Applications and Design (CRC Press, Boca Raton, FL, 2012).

[61] M. M. Radmanesh, Advanced RF \& Microwave Circuit Design (AuthorHouse, Bloomington, 2008).

[62] O. I. Moskalenko, N. S. Phrolov, A. A. Koronovskii, and A. E. Hramov, Eur. Phys. J. Special Topics 222, 2571 (2013).

[63] J. Guittard, S. Penaud, J. C. Nallatamby, and R. Quere, Int. J. RF Microwave Comput-Aided Eng. 12, 469 (2002).

[64] E. V. Efremova, A. Y. Nikishov, and A. I. Panas, in Proceedings of the 5th European Conference on Circuits and Systems for Communications (ECCSC'2010) (IEEE, Belgrade, 2010), p. 67.

[65] A. S. Dmitriev, E. V. Efremova, and N. V. Rumyantsev, Tech. Phys. Lett. 40, 48 (2014).

[66] V. Dronov, M. R. Hendrey, T. M. Antonsen, Jr., and E. Ott, Chaos 14, 30 (2004).

[67] Yu. A. Kalinin, A. A. Koronovskii, A. E. Hramov, E. N. Egorov, and R. A. Filatov, Plasma Phys. Rep. 31, 938 (2005).

[68] A. E. Hramov, A. A. Koronovskii, and S. A. Kurkin, Phys. Lett. A 374, 3057 (2010).

[69] J. H. Choi, editor, High-Speed Devices and Circuits with $\mathrm{THz}$ Applications (CRC Press, Boca Raton, FL, 2015).

[70] J. B. Gunn, IBM J. Res. Dev. 8, 141 (1964).

[71] H. Kroemer, Proc. IEEE 52, 1736 (1964).

[72] J. Benford, J. A. Swegle, and E. Schamiloglu, High Power Microwaves (CRC Press, Boca Raton, FL, 2007).

[73] S. A. Kurkin, A. A. Badarin, A. A. Koronovskii, and A. E. Hramov, Phys. Plasmas. 21, 093105 (2014). 